\title{
Microreactor assisted method for studying isocyanate-alcohol reaction kinetics
}

\author{
Christian Orlando Camacho López ${ }^{1} \cdot$ Zsolt Fejes $^{1}$ (1) $\cdot$ Béla Viskolcz $^{1}$
}

Received: 30 April 2019 / Accepted: 26 June 2019 / Published online: 10 July 2019

(C) The Author(s) 2019

\begin{abstract}
Kinetic parameters of phenyl isocyanate-monoalcohol reactions has been studied using a microreactor system. The monoalcohol components were propan-1-ol, butan-1-ol, propan-2-ol and butan-2-ol. The reactions were conducted in alcohol/THF mixed solvent under pseudo-first-order circumstances at various temperatures. The reaction mixtures were analyzed by off-line HPLC. Reaction rate constants and apparent activation energies have been determined with good precision. The reaction rate constants were higher for the primary alcohols than for the secondary alcohols. Higher apparent activation energy was found for the secondary alcohols compared to the primary ones. The applied technique provides a simple way to study the effects of various factors (e.g. structure of the isocyanate and the alcohol, temperature, solvents, concentration of reagents, catalysis) on the kinetics of the isocyanate-alcohol reactions.
\end{abstract}

Keywords Flow chemistry $\cdot$ Microreactor $\cdot$ Kinetics $\cdot$ Isocyanate $\cdot$ Urethane formation

\section{Introduction}

Microfluidics is a continuously growing field having many application in practically all areas of chemistry, including organic chemistry, chemical technology, biochemistry and also in medicine [1-4]. Advantages of microfluidic devices are rapid and well controlled mixing, precise temperature control, fast and effective mass and heat transfer, reduced reagent consumption and safer conditions [5-7]. Besides optimizing reaction

\section{Highlights}

- Kinetic study on urethane bond formation. Reaction rate constants and activation energies have been determined experimentally.

- Determination of reactivity dependence on alcohol order. Primary alcohols react faster than secondary ones.

- Testing microreactor for urethane formation. The microreactor system proved to be suitable for conducting isocyanate-alcohol reactions.

Zsolt Fejes

zsolt.fejes@gmail.com

1 Institute of Chemistry, Faculty of Materials Science and Engineering, University of Miskolc, Miskolc-Egyetemváros H-3515, Hungary conditions and building compound libraries, combining automation systems with microreactors provide a time and material efficient way to study reaction kinetics. Experiments in series can be performed without the need for preparing new solutions and changing reaction vessels for each experiment, only setting the selected flow rates and temperature are necessary.

The reaction of isocyanates and alcohols is an important transformation regarding not only its synthetic usefulness to prepare carbamates (Scheme 1) but its economical role in producing polyurethane type polymers (Scheme 2). Polyurethanes form as a result of the reaction of an at least difunctional isocyanate with polyols usually containing both primary and secondary hydroxyl groups.

Kinetics of the isocyanate-alcohol reactions have been of interest for many decades. The first detailed investigation was published by Baker and Holdsworth [8]. The uncatalyzed addition reaction follows secondorder kinetics at high alcohol concentrations [9]. Depending on the conditions there may be a deviation from this kinetics at later reaction stages due to the autocatalytic effect exerted by the urethane product [10]. However, the autocatalysis is more pronounced in 


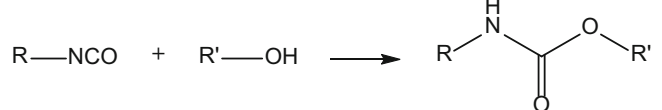

Scheme 1 Reaction of an isocyanate and an alcohol producing a urethane

the case of aliphatic isocyanates [11]. There has been no consensus yet on the reaction mechanism, moreover, it is questionable that a general mechanism for the urethane bond formation exists which gives explanation on the influence of phenomena like catalysis, autocatalysis, solvent effect and molecular interactions on the reaction rate constant and on the activation energy. For a given isocyanate-alcohol system, activation energy dependence on the solvent can be found [12-18]. Some experimental activation energies found in the literature for the uncatalyzed alcoholysis of phenyl isocyanate (PhNCO) using propyl and butyl alcohols are given in Table 1. It can be seen that the values for the primary and secondary alcohols are in the range of 30 $48 \mathrm{~kJ} \mathrm{~mol}^{-1}$ and $41-52 \mathrm{~kJ} \mathrm{~mol}^{-1}$, respectively.

The reactivity of polyols used in polyurethane fabrication is a very important factor and has a large effect on the physical and mechanical properties of the product [19]. As a difference in the reactivity of primary and secondary hydroxyls towards the isocyanate exists, knowing the kinetic parameters (e.g. rate constants and activation energies) can be informative when specific polymer characteristics are needed.

Aim of the present study is to check the applicability of a microreactor system for the rate constant and activation energy determination of the reactions of PhNCO with different primary and secondary alcohols. There has been no study so far in which a microfluidic device is used for kinetic measurements of the alcoholysis of isocyanates.

\section{Results and discussion}

Propan-1-ol, butan-1-ol, propan-2-ol and butan-2-ol were chosen to study their reactions with $\mathrm{PhNCO}$. The microreactor setup is summarized in Fig. 1. 0.04 M PhNCO and 8.0 M alcohol solutions, each prepared in THF, were pumped to the microreactor chip. Because of the equal flow rates, the isocyanate and the alcohol enter the chip at $0.02 \mathrm{M}$ and 4.0 M, respectively. Due to the high alcohol excess, pseudo first order conditions were achieved. At each selected temperature in the range of $40-80^{\circ} \mathrm{C}$, four runs in total were carried out by varying the flow rate of $60-15 \mu \mathrm{L} / \mathrm{min}$ (for each stream) corresponding to $125-500 \mathrm{~s}$ of reaction times. With these conditions, the $\mathrm{PhNCO}$ conversion was in the range of $28-70 \%$ for propan-1-ol, 37-82\% for butan-1-ol and $14-55 \%$ for propan-2-ol and butan-2-ol. The reaction mixture was quenched using $n$-butyl amine which reacts instantaneously and quantitatively with $\mathrm{PhNCO}$ to form the urea derivative $N$-butyl- $N$ '-phenylurea which in turn was quantified by HPLC. Typical HPLC chromatograms are shown in Fig. 2. The minor peak besides the butyl urea and the urethane corresponds to $N, N^{\prime}$-diphenylurea, resulting from the reaction of $\mathrm{PhNCO}$ with the traces of residual moisture present in the alcohols and/or in THF. Quantification of this compound in each run indicated that no more than $5 \%$ of the isocyanate were converted by this side reaction.

Plotting the logarithm of the isocyanate concentration decrease versus the reaction time gave linear relationships for all the studied alcohols (Fig. 3). The maximum relative standard deviation was 5\% (not shown in the figure). For linear fitting, $R^{2}$ values were between 0.996-0.999. The slopes yielded the observed rate constants which in turn were divided by the alcohol concentration to furnish the second-order rate constants (Table 2). The alcohol concentration remains practically constant during the reaction.

The reaction rate constants are 1.4-3 times higher for the primary alcohols than for the secondary ones. Increasing the alcohol chain length results in increased reaction rate difference between the corresponding primary and secondary alcohol. However, this difference is being subsided at higher temperatures. In the case of the propan-1-ol and butan-1-ol, the latter is 1.2-1.4 times more reactive, while the rate constants for propan-2-ol and butan-2-ol are (within the error of measurements) identical.

Apparent activation energies $\left(E_{a}\right)$ and pre-exponential constants $(A)$, shown in Table 2 , were determined using the Arrhenius equation. The Arrhenius plots are shown in Fig. 4. The activation energies are equal within the<smiles>CC(C)(C)OPOC(=O)NNPC(=O)C(C)(C)C(=O)NOPOO[N+](=O)[O-]</smiles>

Scheme 2 Reaction of a diisocyanate and a diol producing a polyurethane 
Table 1 Experimental activation energies of various uncatalyzed $\mathrm{PhNCO}$-monoalcohol reactions

\begin{tabular}{llll}
\hline alcohol & solvent & $E_{a}\left(\mathrm{~kJ} \mathrm{~mol}^{-1}\right)$ & reference \\
\hline 1-propanol & benzene & 36.8 & {$[12]$} \\
1-propanol & $N, N$-dimethylacetamide & 29.9 & {$[13]$} \\
1-propanol & $N, N$-dimethylformamide & 36.5 & \\
2-propanol & 2-propanol & $38.9-41.8$ & {$[14]$} \\
2-propanol & di- $n$-butyl ether & 41.8 & {$[17]$} \\
1-butanol & chlorobenzene & 31 & {$[18]$} \\
1-butanol & xylene & 33.9 & {$[16]$} \\
1-butanol & benzene & 36.8 & {$[12]$} \\
1-butanol & 1-butanol & 48.1 & {$[15]$} \\
2-butanol & 2-butanol & 41.4 & {$[14]$} \\
2-butanol & xylene & 41.4 & {$[16]$} \\
2-butanol & 2-butanol & 52.3 & {$[15]$} \\
\hline
\end{tabular}

error of measurements for the two primary and for the two secondary alcohols, respectively. The activation energy is $30.3 \mathrm{~kJ} \mathrm{~mol}^{-1}$ (average) for propan-1-ol and butan-1-ol and $38.4 \mathrm{~kJ} \mathrm{~mol}^{-1}$ (average) for propan-2-ol and butan-2-ol. The higher energy barrier for the secondary alcohols can be attributed to more steric hindrance of the secondary hydroxyl group compared to a primary hydroxyl. The pre-exponential constants are comparable for both the primary (18.8 and $\left.23.6 \mathrm{~s}^{-1}\right)$ and the secondary (179 and $212 \mathrm{~s}^{-1}$ ) monools, respectively.

\section{Conclusion}

A microreactor technique has been applied for the accurate determination of kinetic parameters of phenyl isocyanate-monoalcohol reactions using THF-alcohol as the solvent. The method is capable to furnish reaction rate constants and activation energies with good precision. As there are many open parameters regarding the elementary steps of the isocyanate-alcohol reactions, our technique provides a simple way to study the effects of various factors (e.g. structure of the isocyanate and the alcohol, temperature, solvents, concentration of reagents, catalysis) on the kinetics of this chemical process. Based on this work, our further aim is to use a microreactor approach for investigating the effect of the solvents and various catalysts on the reaction kinetics in a reliable fashion.

\section{Experimental section}

\section{Chemicals}

Tetrahydrofuran, propan-1-ol, propan-2-ol, butan-1-ol and butan-2-ol (VWR International Ltd.) were analytical grade and stored over $20 \% \mathrm{w} / \mathrm{w}$ activated $3 \AA$ molecular sieves at least for two days, furnishing solvents with no more than $30 \mathrm{ppm}$ of residual water [20]. Acetonitrile (VWR International Ltd.) was chromatography grade. Phenyl isocyanate and $n$-butylamine (Acros Organics) had purity of $99 \%$. $N, N^{\prime}$-diphenylurea (Alfa Aesar) had purity of $\geq 98 \%$. $N$-butyl- $N^{\prime}$-phenylurea, propyl phenylcarbamate, isopropyl phenylcarbamate, butyl phenylcarbamate and sec-butyl phenylcarbamate were prepared by reacting phenyl isocyanate with $n$ butylamine, propan-1-ol, propan-2-ol, butan-1-ol and butan-2-ol, respectively. After purification by flash chromatography on silica gel the products were used for HPLC calibrations.
Fig. 1 Experimental setup of the microreactor system

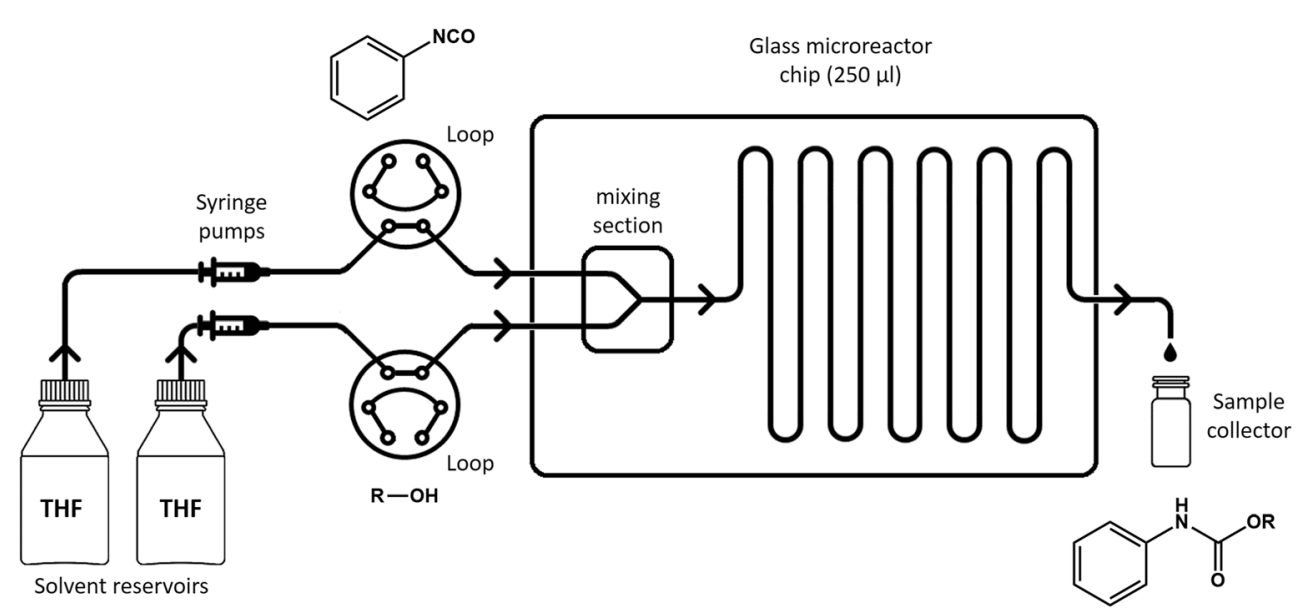




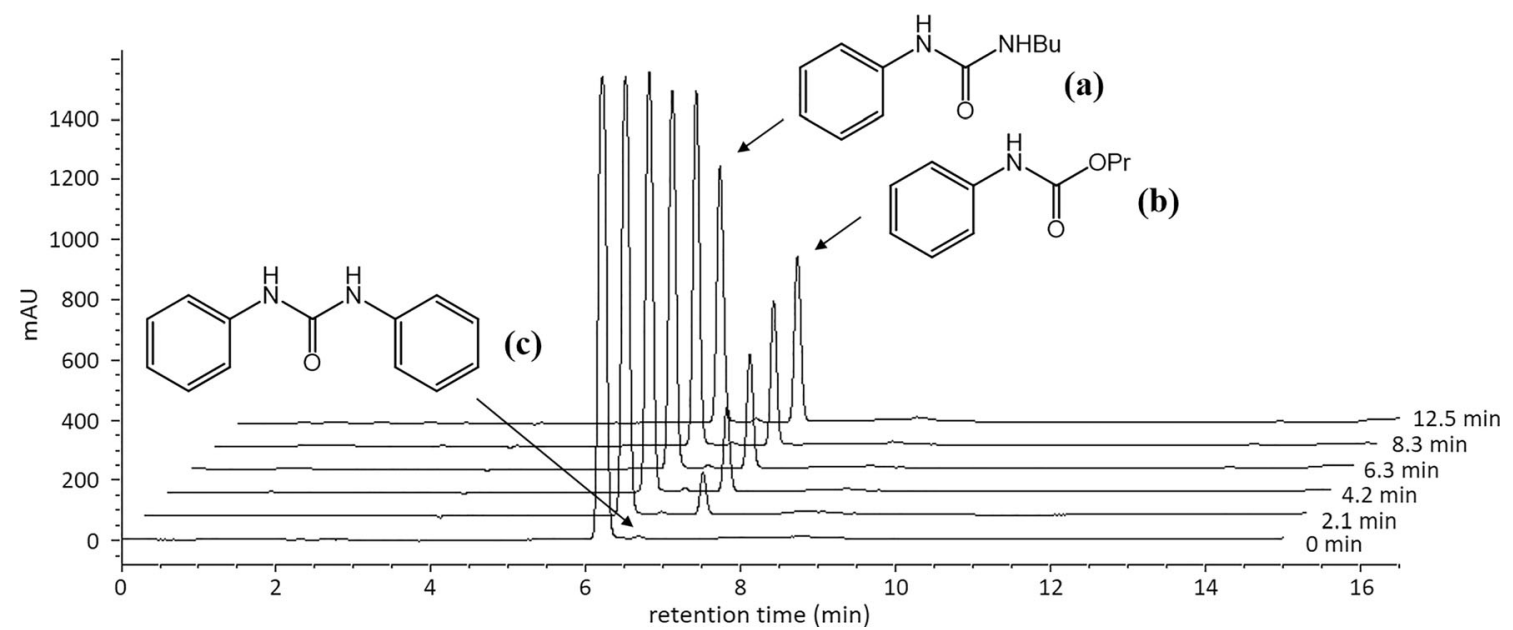

Fig. 2 Typical HPLC chromatograms showing the urea derivative of the non-reacted isocyanate (a), the urethane product of phenyl isocyanate and propan-1-ol (b) and small amount of $N, N^{\prime}$-diphenylurea (c). The different chromatograms correspond to different reaction times, at $60{ }^{\circ} \mathrm{C}$

\section{Microreactor setup}

The Asia microflow system (Syrris Ltd., Royston, UK) consisted of two Asia Syringe Pumps each equipped with 250/500 $\mu \mathrm{L}$ syringe pairs, an Asia Reagent Injector with two $5 \mathrm{~mL}$ loops, an Asia Heater equipped with a microreactor adaptor, an Asia Pressure Controller and an Asia Product Collector. An Asia $250 \mu \mathrm{L}$ glass chip (mixer: $250 \mu \mathrm{m}$ depth, $300 \mu \mathrm{m}$ width, $532 \mathrm{~mm}$ length; microreactor: $250 \mu \mathrm{m}$ depth, $400 \mu \mathrm{m}$ width,
$2509 \mathrm{~mm}$ length) was installed into the microreactor adaptor. Storage bottles for the pumps were filled with THF. The loops were filled with $0.04 \mathrm{M}$ PhNCO solution in THF and $8.0 \mathrm{M}$ alcohol solution in THF, respectively. The flow rates for the two streams were set equal. According to its specification, the maximum standard deviation of the Asia Heater is $\pm 1,3 \%$ in the range of $40-80{ }^{\circ} \mathrm{C}$. The backpressure regulator was set to 10 barg to avoid gas formation (boiling of the solvents) and to guarantee stable and accurate flow rates. The
Fig. 3 Graphs of isocyanate concentration decrease versus time
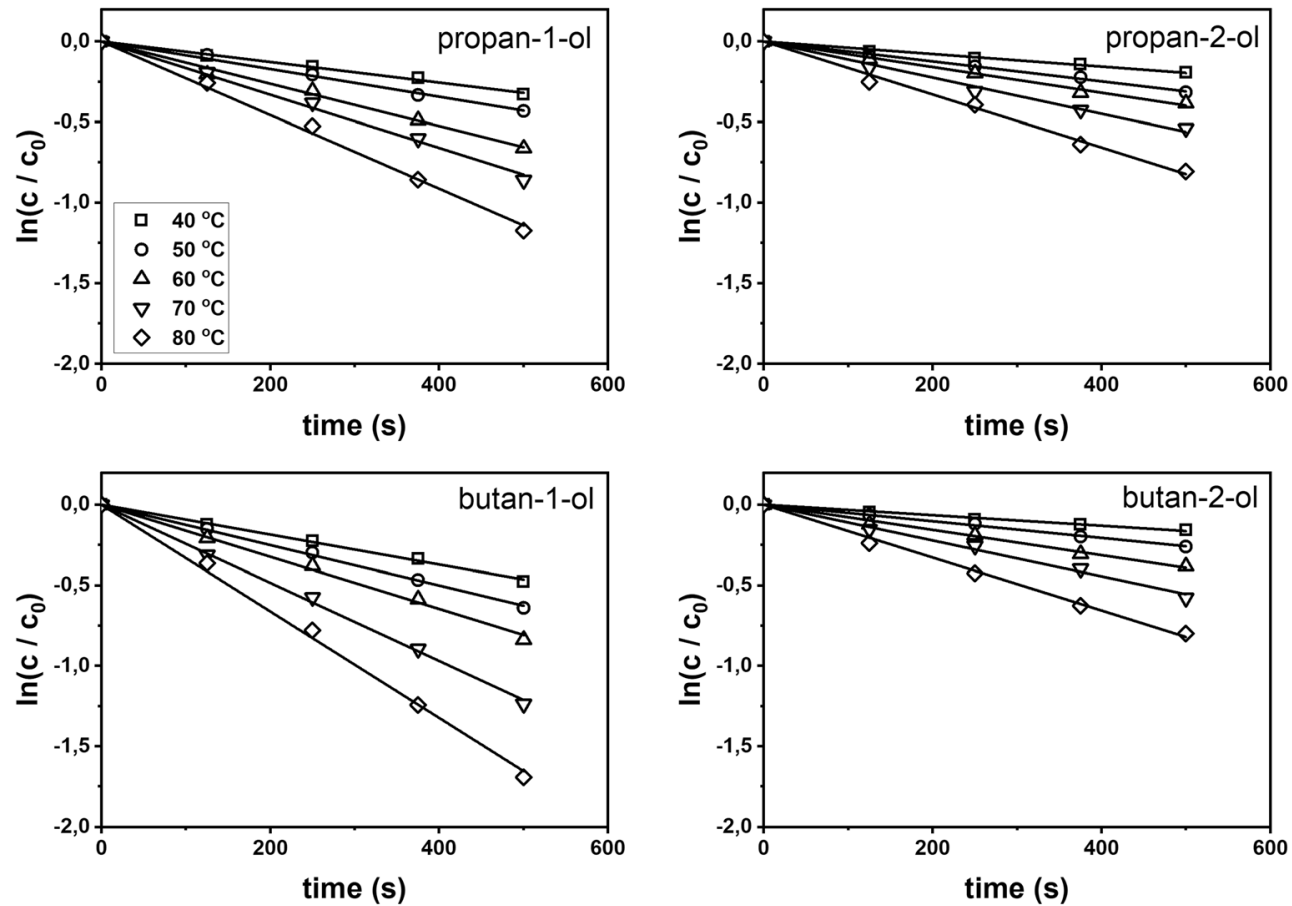
Table 2 Kinetic parameters of the reaction of $\mathrm{PhNCO}$ with monoalcohols. Standard deviation was determined by running five repeated measurements in three selected cases, as indicated in the table

\begin{tabular}{|c|c|c|c|c|c|c|c|}
\hline & \multicolumn{5}{|c|}{$10^{4} k\left(\mathrm{M}^{-1} \mathrm{~s}^{-1}\right)$} & \multirow{2}{*}{$\begin{array}{l}E_{a} \\
\left(\mathrm{~kJ} \mathrm{~mol}^{-1}\right)\end{array}$} & \multirow{2}{*}{$\begin{array}{l}A \\
\left(\mathrm{~s}^{-1}\right)\end{array}$} \\
\hline & $313 \pm 4 \mathrm{~K}$ & $323 \pm 4 \mathrm{~K}$ & $333 \pm 4 \mathrm{~K}$ & $343 \pm 5 \mathrm{~K}$ & $353 \pm 5 \mathrm{~K}$ & & \\
\hline propan-1-ol & $1.55 \pm 0.08$ & 2.34 & 3.28 & 4.45 & 5.85 & 30.4 & 18.8 \\
\hline propan-2-ol & 0.75 & 1.37 & $1.99 \pm 0.08$ & 2.81 & 4.10 & 38.1 & 179 \\
\hline butan-1-ol & 2.22 & 3.13 & 4.04 & 6.06 & $8.27 \pm 0.40$ & 30.2 & 23.6 \\
\hline butan-2-ol & 0.74 & 1.29 & 1.98 & 2.76 & 4.10 & 38.6 & 212 \\
\hline
\end{tabular}

system was operated via the Asia Manager software. The collection volumes were set to $1000 \mu \mathrm{L}$ with minus $250 \mu \mathrm{L}$ pre-slug and minus $250 \mu \mathrm{L}$ post-slug volumes in order to collect only from the steady-state concentration zone of the stream. Practically it means that $500 \mu \mathrm{L}$ of solution was collected into vials containing $1 \mathrm{~mL}$ acetonitrile and $30 \mu \mathrm{L} n$-butylamine. The quenched reaction mixture then was diluted with acetonitrile to $10 \mathrm{~mL}$ in a volumetric flask and subjected to HPLC analysis. The accuracy of the flow rates was verified by pumping $N, N^{\prime}$-dimethyformamide (DMF) through the system at pumping speeds and temperatures used in the study, and collecting volumes of $500 \mu \mathrm{L}$ into pre-weighted vials. Backpressure regulator was set at 10 barg. DMF was chosen as being non-volatile there is practically no evaporation during the collection period. Weighting the collected volumes using analytical balance indicated the flow rates to be within $\pm 0,5 \%$ in each flow rate-temperature set.

\section{Analysis method}

PhNCO concentrations were determined by HPLC via the urea derivative $N$-butyl- $N$ '-phenylurea. The analysis was performed using a HP 1050 equipped with a LiChroCART 250-4 LiChrospher 100 RP-18 column $(4 \times 250 \mathrm{~mm}, 5 \mu \mathrm{m})$ and an ODS-Hypersil C18 guard column $(2.1 \times 20 \mathrm{~mm}, 5 \mu \mathrm{m})$, both operated at $40{ }^{\circ} \mathrm{C}$. Samples were injected via autosampler from a $20 \mu \mathrm{L}$ sample loop. Compounds were detected and quantified
Fig. 4 Temperature dependence of the reaction rate constants
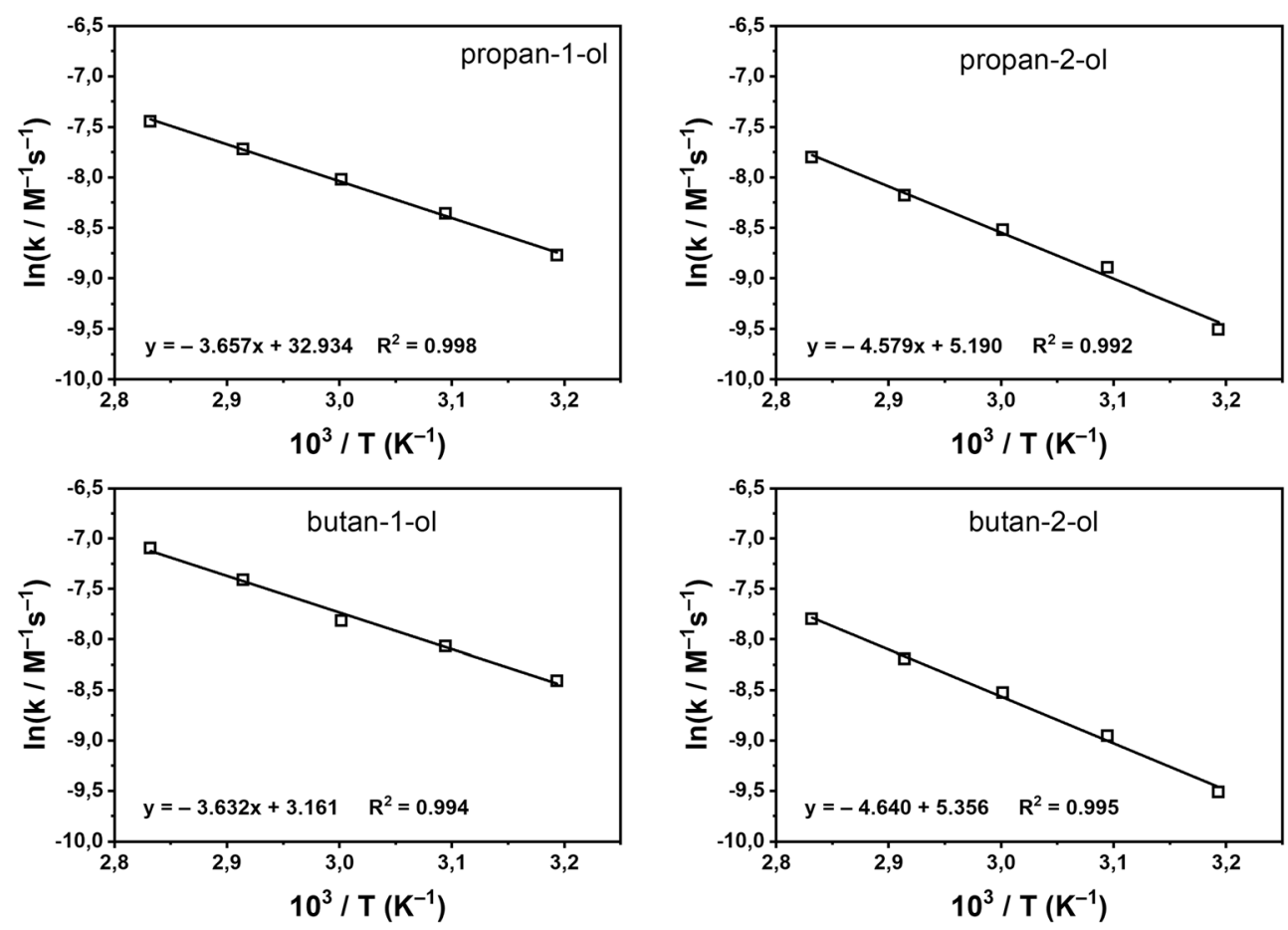
with a UV absorbance detector at $246 \mathrm{~nm}$. The mobile phase consisted of water and acetonitrile. The gradient conditions were as follows: $0 \mathrm{~min}, 25 \%$ acetonitrile; 0 $6 \mathrm{~min}, 25 \%$ to $80 \%$ acetonitrile; $6-10 \mathrm{~min}, 80 \%$ acetonitrile; $10-11 \mathrm{~min}, 80 \%$ to $25 \%$ acetonitrile; $11-14 \mathrm{~min}$, $25 \%$ acetonitrile (re-equilibration) at a flow rate of $1 \mathrm{~mL} / \mathrm{min}$.

Acknowledgements Open access funding provided by University of Miskolc (ME). This research was supported by the European Union and the Hungarian State, co-financed by the European Regional Development Fund in the framework of the GINOP-2.3.4-15-201600004 project, aimed to promote the cooperation between the higher education and the industry.

Open Access This article is distributed under the terms of the Creative Commons Attribution 4.0 International License (http:// creativecommons.org/licenses/by/4.0/), which permits unrestricted use, distribution, and reproduction in any medium, provided you give appropriate credit to the original author(s) and the source, provide a link to the Creative Commons license, and indicate if changes were made.

\section{References}

1. Mason BP, Price KE, Steinbacher JL, Bogdan AR, McQuade DT (2007). Chem Rev 107:2300-2318

2. Šalić A, Tušek A, Zelić B (2012). J Appl Biomed 10:137-153

3. Chiu DT, deMello AJ, Di Carlo D, Doyle PS, Hansen C, Maceiczyk RM, Wootton RCR (2017). Chem. 2:201-223
4. Livak-Dahl E, Sinn I, Burns M (2011). Annu Rev Chem Biomol Eng 2:325-380

5. Ehrfeld W, Hessel V, Löwe H (2000) Microreactors: new Technology for Modern Chemistry. Wiley-VCH, Weinheim

6. Jähnisch K, Hessel V, Löwe H, Baerns M (2004). Angew Chem Int Ed 43:406-446

7. Fanelli F, Parisi G, Degennaro L, Luisi R (2017). Beilstein J Org Chem 13:520-542

8. Baker JW, Holdsworth JB (1947) J Chem Soc 0:713-726

9. Jones FN, Nichols ME, Pappas SP (2017) Organic coatings: science and technology (4th edn.) John Wiley \& Sons Inc

10. Cordeiro N, Belgacem MN, Gandini A, Neto CP (1997). Ind Crop Prod 6:163-167

11. Sato M (1960). J Am Chem Soc 82:3893-3897

12. Sivakamasundari S, Ganesan R (1981). J. Org. Chem. 49:720-722

13. Yang PF, Yu YH, Li TD, Zhang M (2013). Int J Polym Anal Ch 18: $57-63$

14. Xu L, Li C, Ng S, Ng KY, ( (2000). J Phys Chem A 104:3952-3957

15. Lovering EG, Laidler KJ (1962). Can J Chem 40:31-36

16. Dyer E, Taylor HA, Masonand SJ, Sams J (1949). J Am Chem Soc 71:4106-4109

17. Baker JW, Gaunt J (1949). J Chem Soc 0:19-24

18. Samuilov AY, Samuilov YD (2018). Russ J Org Chem 54:17491753

19. Szycher M (2012) Szycher's handbook of polyurethanes (2nd edn.) CRC Press, Taylor \& Francis Group, Boca Raton

20. Williams DBG, Lawton M (2010). J Org Chem 75:8351-8354

Publisher's note Springer Nature remains neutral with regard to jurisdictional claims in published maps and institutional affiliations. 